\title{
Adversarial Open-World Person Re-Identification
}

\author{
Xiang $\mathrm{Li}^{1}$, Ancong $\mathrm{Wu}^{1}$, and Wei-Shi Zheng ${ }^{1,2,3 *[0000-0001-8327-0003]}$ \\ 1 Sun Yat-sen University \\ \{lixiang47, wuancong $\} @$ mail2. sysu.edu.cn \\ wszheng@ieee.org \\ 2 Inception Institute of Artificial Intelligence, United Arab Emirates \\ 3 Key Laboratory of Machine Intelligence and Advanced Computing, MOE
}

\begin{abstract}
In a typical real-world application of re-id, a watch-list (gallery set) of a handful of target people (e.g. suspects) to track around a large volume of non-target people are demanded across camera views, and this is called the open-world person re-id. Different from conventional (closedworld) person re-id, a large portion of probe samples are not from target people in the open-world setting. And, it always happens that a nontarget person would look similar to a target one and therefore would seriously challenge a re-id system. In this work, we introduce a deep open-world group-based person re-id model based on adversarial learning to alleviate the attack problem caused by similar non-target people. The main idea is learning to attack feature extractor on the target people by using GAN to generate very target-like images (imposters), and in the meantime the model will make the feature extractor learn to tolerate the attack by discriminative learning so as to realize group-based verification. The framework we proposed is called the adversarial open-world person re-identification, and this is realized by our Adversarial PersonNet (APN) that jointly learns a generator, a person discriminator, a target discriminator and a feature extractor, where the feature extractor and target discriminator share the same weights so as to makes the feature extractor learn to tolerate the attack by imposters for better group-based verification. While open-world person re-id is challenging, we show for the first time that the adversarial-based approach helps stabilize person re-id system under imposter attack more effectively.
\end{abstract}

\section{Introduction}

Person re-identification (re-id), which is to match a pedestrian across disjoint camera views in diverse scenes, is practical and useful for many fields, such as public security applications and has gained increasing interests in recent years 3. $3,6,10,11,22,34,36,37,40,42,45$. Rather than re-identifying every person in a multiple camera network, a typical real-world application is to re-identify or track only a handful of target people on a watch list (gallery set), which is called

*corresponding author 


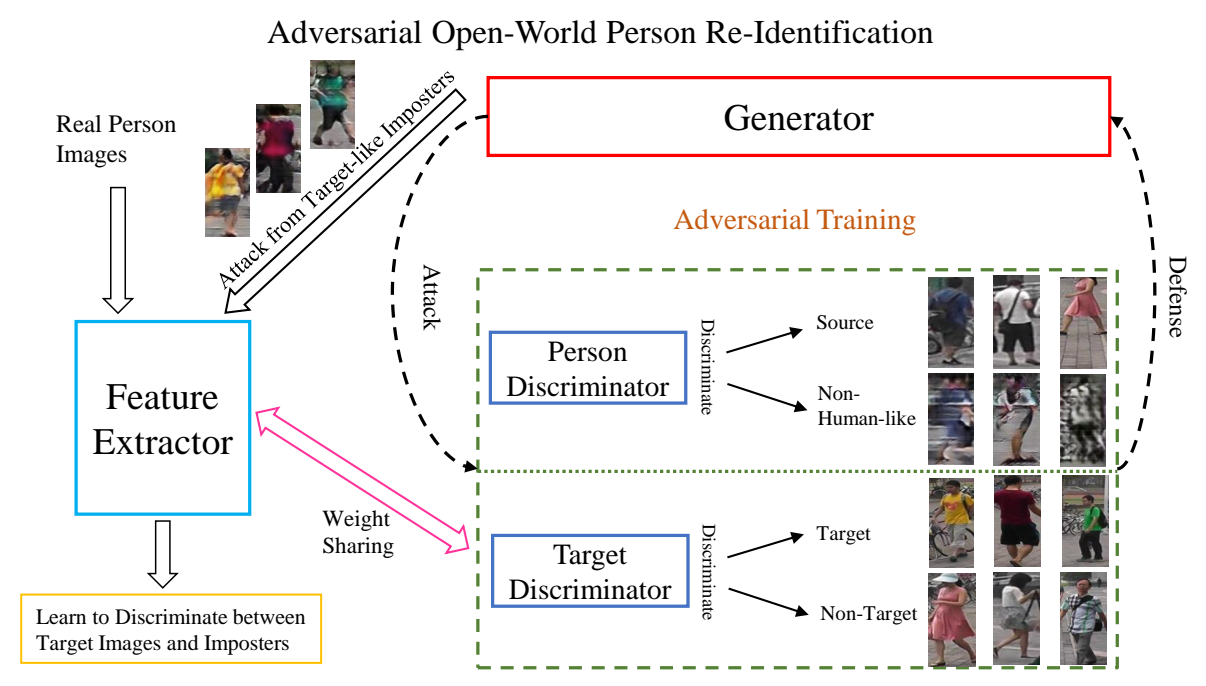

Fig. 1: Overview of adversarial open-world person re-identification. The goal for the generator is to generate target-like images, while we have two discriminators here. The person discriminator is to discriminate whether the generated images are from source dataset (i.e. being human-like). And the target discriminator is to discriminate whether the generated images are of target people. By the adversarial learning, we aim to generate images beneficial for training a better feature extractor for telling target person images apart from non-target ones.

the open-world person re-id problem $4,42,46$. While target people will reappear in the camera network at different views, a large volume of non-target people, some of which could be very similar to target people, would appear as well. This contradicts to the conventional closed-world person re-id setting that all probe queries are belonging to target people on the watch list. In comparison, the open-world person re-id is extremely challenging because both target and non-target (irrelevant) people are included in the probe set.

However, the majority of current person re-identification models are designed for the closed-world setting $66,33,36,38,40,43,45]$ rather than the open-world one. Without consideration of discriminating target and non-target people during learning, these approaches are not stable and could often fail to reject a query image whose identity is not included in the gallery set. Zheng et al. 42 considered this problem and proposed open-world group-based verification model. Their model is based on hand-crafted feature and transfer-learning-based metric learning with auxiliary data, but the results are still far from solving this challenge. More importantly, the optimal feature representation and target-personspecific information for open-world setting have not been learned.

In this work, we present an adversarial open-world person re-identification framework for 1) learning features that are suitable for open-world person re-id, 
and 2) learning to attack the feature extractor by generating very target-like imposters and make person re-id system learn to tolerate it for better verification. An end-to-end deep neural network is designed to realize the above two objectives, and an overview of this pipeline is shown in Fig. 11. The feature learning and the adversarial learning are mutually related and learned jointly, meanwhile the generator and the feature extractor are learned from each other iteratively to enhance both the efficiency of generated images and the discriminability of the feature extractor. To use the unlabeled images generated, we further incorporate a label smoothing regularization for imposters (LSRI) for this adversarial learning process. LSRI allocates equal probabilities of being any non-target people and zero probabilities of being target people to the generated target-like imposters, and it would further improve the discrimination ability of the feature extractor for distinguishing real target people from fake ones (imposters).

While GAN has been attempted in Person re-id models recently in 8, 43,44 for generating images adapted from source dataset so as to enrich the training dataset on target task. However, our objective is beyond this conventional usage. By sharing the weights between feature extractor and target discriminator (see Fig. 2), our adversarial learning makes the generator and feature extractor interact with each other in an end-to-end framework. This interaction not only makes the generator produce imposters look like target people, but also more importantly makes the feature extractor learn to tolerate the attack by imposters for better group-based verification.

In summary, our contributions are more on solving the open-world challenge in person re-identification. It is the first time to formulate the open-world group-based person re-identification under an adversarial learning framework. By learning to attack and learning to defend, we realize four progresses in a unified framework, including generating very target-like imposters, mimicking imposter attacking, discriminating imposters from target images and learning re-id feature to represent. Our investigation suggests that adversarial learning is a more effective way for stabilizing person re-id system undergoing imposters.

\section{Related Work}

Person Re-Identification: Since person re-identification targets to identify different people, better feature representations are studied by a great deal of recent research. Some of the research try to seek more discriminative/reliable hand-crafted features $[10,17,22,23,25,26,37$. . Except that, learning the best matching metric $[5,6,14,18,27,33,40$ is also widely studied for solving the cross-view change in different environments. With the rapid development of deep

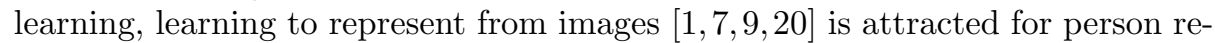
id, and in particular Xiao et al. 38 came up with domain guided drop out model for training CNN with multiple domains so as to improve the feature learning procedure. Also, recent deep approaches in person re-identification are found to unify feature learning and metric learning $1,31,36,45$. Although these deep learning methods are expressive for large-scale datasets, they tend to be resistless 
for noises and incapable of distinguishing non-target people apart from the target ones, and thus becomes unsuitable for the open-world setting. In comparison, our deep model aims to model the effect of non-target people during training and optimize the person re-id in the open-world setting.

Towards Open-World Person Re-Identification: Although the majority of works on person re-id are focusing on the closed-world setting, a few works have been reported on addressing the open-world setting. The work of Candela et al. [4] is based on Conditional Random Field (CRF) inference attempting to build connections between cameras towards open-world person re-identification. But their work lacks the ability to distinguish very similar identities, and with some deep CNN models coming up, features from multiple camera views can be well expressed by joint camera learning. Wang et al. 34] worked out an approach by proposing a new subspace learning model suitable for open-world scenario. However, group-based setting and interference defense is not considered. Also, their model requires a large volume of extra unlabeled data. Zhu et al. 46] proposed a novel hashing method for fast search in the open-world setting. However, Zhu et al. aimed at large scale open-world re-identification and efficiency is considered primarily. Besides, noiseproof ability is not taken into account. The most correlated work with this paper is formulated by Zheng et al. [41, 42, where the group-based verification towards open-world person re-identification was proposed. They came up with a transfer relative distance comparison model (t-LRDC), learning a distance metric and transferring non-target data to target data in order to overcome data sparsity. Different from the above works, we present the first end-to-end learning model to unify feature learning and verification modeling to address the open-world setting. Moreover, our work does not require extra auxiliary datasets to mimic attack of imposters, but integrates an adversarial processing to make re-id model learn to tolerate the attack.

Adversarial Learning: In 2014, Szegedy et al. 32] have found out that tiny noises in samples can lead deep classifiers to mis-classify, even if these adversarial samples can be easily discriminated by human. Then many researchers have been working on adversarial training. Seyed-Mohsen et al. [28] proposed DeepFool, using the gradient of an image to produce a minimal noise that fools deep networks. However, their adversarial samples are towards individuals and the relation between target and non-target groups is not modelled. Thus, it does not well fit into the group-based setting. Nicolas Papernot et al. [29 formulated a class of algorithms by using knowledge of deep neural networks (DNN) architecture for crafting adversarial samples. However, rather than forming a general algorithm for DNNs, our method is more specific for group-based person verification and the imposter samples generated are more effective to this scenario. Later, SafetyNet by Lu et al. 24] was proposed with an RBF-SVM in full-connected layer to detect adversarial samples. However, we perform the adversarial learning at feature level to better attack the learned features. 


\section{Adversarial PersonNet}

\subsection{Problem Statement}

In this work, we concentrate on open-world person re-id by group-based verification. The group-based verification is to ensure a re-id system to identify whether a query person image comes from target people on the watch list. In this scenario, people out of this list/group are defined as non-target people.

Our objective is to unify feature learning by deep convolution networks and adversarial learning together so as to make the extracted feature robust and resistant to noise for discriminating between target people and non-target ones. The adversarial learning is to generate target-like imposter images to attack the feature extraction process and simultaneously make the whole model learn to distinguish these attacks. For this purpose, we propose a novel deep learning model called Adversarial PersonNet (APN) that suits open-world person re-id.

To better express our work under this setting in the following sections, we suppose that $N_{T}$ target training images constitute a target sample set $X_{T}$ sampled from $C_{T}$ target people. Let $\boldsymbol{x}_{i}^{T}$ indicate the $i$ th target image and $y_{i}^{T} \in Y_{T}$ represents the corresponding person/class label. The label set $Y_{T}$ is denoted by $Y_{T}=\left\{y_{1}^{T}, \ldots, y_{N_{T}}^{T}\right\}$ and there are $C_{T}$ target classes in total. Similarly, we are given a set of $C_{S}$ non-target training classes containing $N_{S}$ images, denoted as $X_{S}=\left\{\boldsymbol{x}_{1}^{S}, \ldots, \boldsymbol{x}_{N_{S}}^{S}\right\}$, where $\boldsymbol{x}_{i}^{S} \in X_{S}$ is the $i$ th non-target image. $y_{i}^{S}$ is the class of $\boldsymbol{x}_{i}^{S}$ and $Y_{S}=\left\{y_{1}^{S}, \ldots, y_{N_{S}}^{S}\right\}$. Note that there is no identity overlap between target people and non-target people. Under open-world setting, $N_{S} \gg N_{T}$. The problem is to better determine whether a person is on the target-list; that is for a given image $\boldsymbol{x}$ without knowing its class $y$, determine if $y \in Y_{T}$. We use $f(\boldsymbol{x}, \boldsymbol{\theta})$ to represent the extracted feature from image $\boldsymbol{x}$, and $\boldsymbol{\theta}$ is the weight of the feature extraction part of the CNN.

\subsection{Learning to Attack by Adversarial Networks}

Always, GANs are designed to generate images similar to those in the source set, which is constituted by both target and non-target image sets. A generator $G$ and a discriminator $D_{p}$ are trained adversarially. However the generator $G$ normally only generates images looking like the ones in the source set and the discriminator $D_{p}$ discriminates the generated images from the source ones. In this case, our source datasets are all pedestrian images, so we call such $D_{p}$ the person

discriminator in response to its ability of determining whether an image is of pedestrian-like images. $D_{p}$ is trained by minimizing the following loss function:

$$
L_{D_{p}}=-\frac{1}{m} \sum_{i=1}^{m}\left[\log D_{p}(\boldsymbol{x})+\log \left(1-D_{p}(G(\boldsymbol{z}))\right)\right],
$$

where $m$ is the number of samples, $\boldsymbol{x}$ represents image from source dataset and $\boldsymbol{z}$ is a noise randomly generated. 


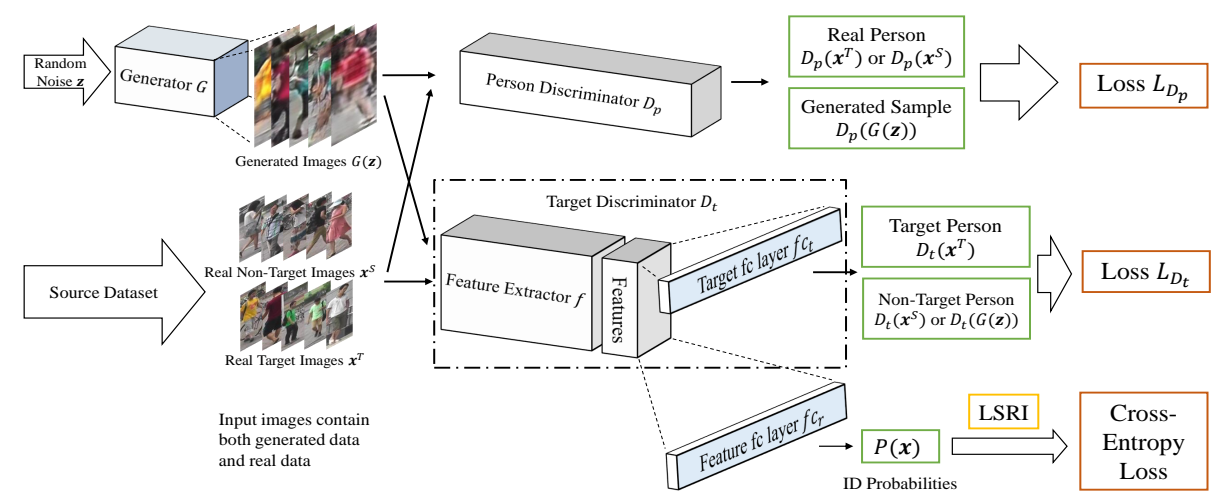

Fig. 2: Adversarial PersonNet structure. Two discriminators $D_{p}$ and $D_{t}$ accept samples from both datasets and generator $G$. Since $D_{t}$ shares the same weights with feature extractor $f$, we represent them as the same cuboid in this figure.

Suppose that there is a pre-trained feature extractor for person re-id task and in an attempt to steer generator $G$ to produce not only pedestrian-like but also feature attacking images towards this feature extractor, we design a paralleled discriminator $D_{t}$ with the following definition:

$$
D_{t}(\boldsymbol{x})=f c_{t}(f(\boldsymbol{x}, \boldsymbol{\theta})) .
$$

The discriminator $D_{t}$ is to determine whether an image will be regarded as target image by feature extractor. $f(\boldsymbol{x}, \boldsymbol{\theta})$ indicates that part of $D_{t}$ has the same network structure as feature extractor $f$ and shares the same weights $\boldsymbol{\theta}$ (Actually, the feature extractor can be regarded as a part of $D_{t}$.). $f c_{t}$ means a full-connected layer following the feature extractor apart from the one connected to original CNN (with a fc layer used to pre-train the feature extractor). So $D_{t}$ shares the same ability of target person discrimination with the feature extractor. To induce the generator $G$ for producing target-like images for attacking and ensure the discriminator $D_{t}$ to tell the non-target and generated imposters apart from the target ones, we formulate a paralleled adversarial training of $G$ and $D_{t}$ as

$$
\begin{aligned}
\min _{G} \max _{D_{t}} V_{t}\left(D_{t}, G\right) & =\mathbb{E}_{\boldsymbol{x}^{T} \sim X_{T}}\left[\log D_{t}\left(\boldsymbol{x}^{T}\right)\right] \\
& +\mathbb{E}_{\boldsymbol{x}^{S} \sim X_{S}}\left[\log \left(1-D_{t}\left(\boldsymbol{x}^{S}\right)\right)\right] \\
& +\mathbb{E}_{\boldsymbol{z} \sim p_{\boldsymbol{z}}(\boldsymbol{z})}\left[\log \left(1-D_{t}(G(\boldsymbol{z}))\right)\right] .
\end{aligned}
$$

We train $D_{t}$ to maximize $D_{t}(\boldsymbol{x})$ when passed by a target image but minimize it when passed by a non-target image or a generated imposter image by $G$. Notice that this process only trains the final $f c_{t}$ layer of $D_{t}$ without updating the feature extractor weights $\boldsymbol{\theta}$, to prevent the feature extractor from being affected by discriminator learning when the generated images are not good enough. We 


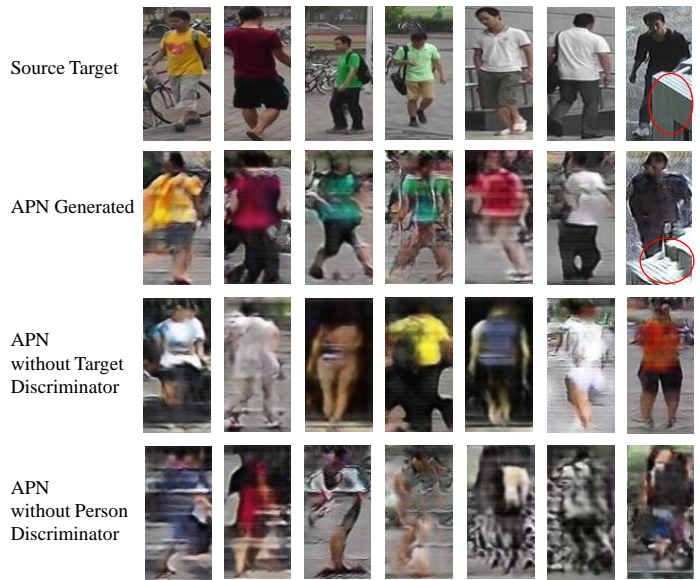

Fig. 3: Examples of generated images. Although images produced by the generator are based on random noises, we can tell that the imposters generated by APN are very similar to targets. These similarities are mostly based on clothes, colors and postures (e.g. the fifth column). Moreover, surroundings are learned by APN as shown in the seventh column in the red circle.

call $D_{t}$ the target discriminator. And we propose the loss function $L_{D_{t}}$ for the training process of target discriminator $D_{t}$ :

$$
\left\{\begin{array}{l}
L_{D_{t}}=-\frac{1}{m} \sum_{i=1}^{m}\left[\log Q_{t}(\boldsymbol{x})+\log \left(1-D_{t}(G(\boldsymbol{z}))\right)\right], \\
Q_{t}(\boldsymbol{x})= \begin{cases}D_{t}(\boldsymbol{x}), & \boldsymbol{x} \in X_{T} \\
1-D_{t}(\boldsymbol{x}), & \boldsymbol{x} \in X_{S}\end{cases}
\end{array}\right.
$$

We integrate the above into a standard GAN framework as follows:

$$
\begin{aligned}
& \min _{G} \max _{D_{p}} \max _{D_{t}} V^{\prime}\left(D_{p}, D_{t}, G\right)= \\
& \mathbb{E}_{\boldsymbol{x}^{T} \sim X_{T}}\left[\log D_{p}\left(\boldsymbol{x}^{T}\right)+\log D_{t}\left(\boldsymbol{x}^{T}\right)\right] \\
& +\mathbb{E}_{\boldsymbol{x}^{S} \sim X_{S}}\left[\log D_{p}\left(\boldsymbol{x}^{S}\right)+\log \left(1-D_{t}\left(\boldsymbol{x}^{S}\right)\right)\right] \\
& +\mathbb{E}_{\boldsymbol{z} \sim p_{\boldsymbol{z}}(\boldsymbol{z})}\left[\log \left(1-D_{p}(G(\boldsymbol{z}))\right)+\log \left(1-D_{t}(G(\boldsymbol{z}))\right)\right] .
\end{aligned}
$$

The collaboration of generator and couple discriminators is illustrated in Fig. 2. While GAN with only person discriminator will force the generator $G$ to produce source-like person images, with the incorporation of the loss of target discriminator $D_{t}, G$ is more guided to produce very much target-like imposter images. The target-like imposters, generated based on the discriminating ability of feature extractor, satisfy the usage of attacking the feature extractor. Examples of images generated by APN are shown in Fig. 3 together with the target images and the images generated by controlled groups (APN without target discriminator $D_{t}$ and APN without person discriminator $\left.D_{p}\right)$ to indicate that our network indeed has the ability to generate target-like images. The generator $G$ is trained to fool the target discriminator in the feature space, so that the generated adversarial images can attack the re-id system. While the target discriminator $D_{t}$ is mainly to tell these attack apart from the target people so as to defend the re-id system. 


\subsection{Joint Learning of Feature Representation and Adversarial Modelling}

We finally aim to learn robust person features that are tolerant to imposter attack for open-world group-based person re-id. For further utilizing the generated person images to enhance the performance, we jointly learn feature representation and adversarial modelling in a semi-supervised way.

Although the generated images look similar to target images, they are regarded as imposter samples, and we wish to incorporate unlabeled generated imposter samples. Inspired by the smoothing regularization [43, we modify the LSRO 43 in order to make it more suitable for group-based verification by setting the probability of an unlabeled generated imposter sample $G(\boldsymbol{z})$ belonging to an existing known class $k$ as follows:

$$
q_{L S R I}(k)(G(\boldsymbol{z}))= \begin{cases}\frac{1}{C_{S}}, & k \in Y_{S} \\ 0, & k \in Y_{T},\end{cases}
$$

Compared to LSRO, we do not allocate a uniform distribution on each unlabeled data sample over all classes (including both target and non-target ones), but only allocate a uniform distribution on non-target classes. This is significant because we attempt to separate imposter samples from target classes. The modification is exactly for the defense towards the attack of imposter samples. By using this regularization, the generated imposters are more trending to be far away from target classes and have equal chances of being non-target. We call the modified regularization in Eq. (6) as label smoothing regularization for imposters (LSRI).

Hence for each input sample $\boldsymbol{x}_{i}$, we set its ground truth class distribution as:

$$
q(k)= \begin{cases}1, & k=y_{i} \text { and } \boldsymbol{x}_{i} \in X_{T} \cup X_{S} ; \\ 0, & k \neq y_{i} \text { and } \boldsymbol{x}_{i} \in X_{T} \cup X_{S}, \text { or } \boldsymbol{x}_{i} \in X_{G} \text { and } k \in Y_{T} ; \\ \frac{1}{C_{S}}, & \boldsymbol{x}_{i} \in X_{G}, \text { and } k \in Y_{S} ;\end{cases}
$$

where $y_{i}$ is the corresponding label of $x_{i}$, and we let $\boldsymbol{x}_{i}^{G}$ be the $i$ th generated image and denote $X_{G}=\left\{\boldsymbol{x}_{1}^{G}, \ldots, \boldsymbol{x}_{N_{G}}^{G}\right\}$ as the set of generated imposter samples. With Eq. (7), we can now learn together with our feature extractor (i.e. weights $\boldsymbol{\theta}$ ). By such a joint learning, the feature learning part will become more discriminative between target and target-like imposter images.

\subsection{Network Structure}

We now detail the network structure. As shown in Fig. 2, our network consists of two parts: 1) learning robust feature representation, and 2) learning to attack by adversarial networks. For the first part, we train the feature extractor from source datasets and generated attacking samples. In this part, features are trained to be robust and resistant to imposter samples. LSRI is applied in this part to differentiate imposters from target people. Here, a full-connected layer $f c_{r}$ is connected to feature extractor $f$ at this stage, and we call it the feature $f c$ 
layer. For the second part, as shown in Fig. 2, our learning attack by adversarial networks is a modification of DCGAN 36]. We combine modified DCGAN with couple discriminators to form an adversarial network. The generator $G$ here is modified to produce target-like imposters specifically as an attacker. And the target discriminator $D_{t}$ defends as discriminating target from non-target people. Of course, in this discriminator, a new $f c$ layer is attached to the tail of feature extractor $f$, and we mark it $f c_{t}$, also called target $f c$ layer, used to discriminate target from non-target images at the process of learning to attack by adversarial networks. By Eq. (2), $D_{t}$ is the combination of $f$ and target fc layer $f c_{t}$.

\section{Experiments $4^{4}$}

\subsection{Group-based Verification Setting}

We followed the criterion defined in 42 for evaluation of open-world group-based person re-id. The performance of how well a true target can be verified correctly and how badly a false target can be verified as true incorrectly is indicated by true target rate (TTR) and false target rate (FTR), which are defined as follows:

True Target Rate $(\mathbf{T T R})=\# T T Q / \# T Q$, False Target Rate $(\mathbf{F T R})=\# F N T Q / \# N T Q$,

where $T Q$ is the set of query target images from target people, $N T Q$ is the set of query non-target images from non-target people, $T T Q$ is the set of query target images that are verified as target people, and $F N T Q$ is the set of query non-target images that are verified as target people.

To obtain TTR and FTR, we follow the two steps below: 1) For each target person, there is a set of images $S$ (single-shot or multi-shot) in gallery set. Given a query sample $\boldsymbol{x}$, the distance between sample $\boldsymbol{x}$ and a set $S$ is the minimal distance between that sample and any target sample of that set; 2) Whether a query image is verified as a target person is determined by comparing the distance to a threshold $r$. By changing the threshold $r$, a set of TTR and FTR values can be obtained. A higher TTR value is preferred when FTR is small.

In our experiments, we conducted two kinds of verification as defined in 42 namely Set Verification (i.e. whether a query is one of the persons in the target set, where the target set contains all target people.) and Individual Verification (i.e. whether a query is the true target person. For each target query image, the target set contains only this target person). In comparison, Set Verification is more difficult. Although determining whether a person image belongs to a group of target people seems easier, it also gives more chances for imposters to cheat the classifier, producing more false matchings [42]. Also note that it is more important to compare the TTR values when FTR is low since it is expected to have more true matching when the false matching is less.

\footnotetext{
${ }^{4}$ We correct a bug in our code and conduct a better finetune using new implementations in PyTorch for all deep learning methods including ours and the compared ones. Our model is still effective and overall the best. Please refer to the new results.
} 


\subsection{Datasets \& Settings}

We evaluated our method on three datasets including Market-1501 39], CUHK01 [19], and CUHK03 21]. For each dataset, we randomly selected $1 \%$ people as target people and the rest as non-target. Similar to [42, for target people, we separated images of each target people into training and testing sets by half. Since only four images are available in CUHK01, we chose one for training, two for gallery (reduce to one in single-shot case) and one for probe. Our division guaranteed that probe and gallery images are from diverse cameras for each person. For non-target people, they were divided into training and testing sets by half in person/class level to ensure there is no overlap on identity. In testing phase, two images of each target person in testing set were randomly selected to form gallery set, and the remaining images were selected to form query set. In the default setting, all images of non-target people in testing set were selected to form query set. The data split was kept the same for all evaluations on our and the compared methods. Specifically, the data split is summarized below:

CUHK01 CUHK01 contains 3,884 images of 971 identities from two camera views. In our experiment, 9 people were marked as target and 1,888 images of 472 people were selected to to form the non-target training set. The testing set of non-target people contains 1,960 images of 490 people.

CUHK03 CUHK03 is larger than CUHK01 and some images were automatically detected. A total of 1,467 identities were divided into 14 target people, 712 training non-target people and 741 testing non-target people. The numbers of training and testing non-target images were 6,829 and 7,129 respectively.

Market-1501 Market-1501 is a large-scale dataset containing a total of 32,668 images of 1,501 identities. We randomly selected 15 people as target and 728 people as non-target to form the training set containing a total of 17,316 images, and the testing non-target set contains 758 identities with 14,573 images.

Under the above settings, we evaluated our model together with selected popular re-id models. We used the traditional hand-crafted features suggested in their original paper ${ }^{5}$ while evaluating metric learning methods such as tLRDC [42], XICE [46, XQDA 22] and CRAFT [6].

\subsection{Implementation Details}

In our APN, we used ResNet-50 [12] as the feature extractor in the target discriminator. The generator and person discriminator is based on DCGAN [30]. At the first step of our procedure, we pre-trained the feature extractor using auxiliary datasets, 3DPeS [2], iLIDS [35], PRID2011 [13] and Shinpuhkan [15]. These datasets were only used in the pre-training stage for the feature extractor.

\footnotetext{
${ }^{5}$ We applied these metric learning methods to features extracted by ResNet-50 in the previous version to show our improvement on ResNet-50, but we found that without combining the whole process as an end-to-end learning, the performance of ResNet-50 feature based metric learning methods will largely decrease.
} 
Table 1: Comparison with typical person re-identification: TTR (\%) against FTR

\begin{tabular}{|c|c|c|c|c|c|c|c|c|c|c|c|c|c|c|c|c|c|c|}
\hline \multirow{2}{*}{$\begin{array}{c}\text { Dataset } \\
\text { FTR }\end{array}$} & \multicolumn{6}{|c|}{ Market-1501 } & \multicolumn{6}{|c|}{ CHUK01 } & \multicolumn{6}{|c|}{ CUHK03 } \\
\hline & $\%$ & $1 \%$ & $5 \%$ & $10 \%$ & $20 \%$ & $30 \%$ & $0.1 \%$ & $1 \%$ & $5 \%$ & $10 \%$ & $20 \%$ & $30 \%$ & $1 \%$ & $1 \%$ & $5 \%$ & $10 \%$ & $20 \%$ & $30 \%$ \\
\hline Evaluation & \multicolumn{18}{|c|}{ Set Verification } \\
\hline t-LRDC 42] & 00 & 18.88 & 42.06 & 51.07 & 65.24 & 75.54 & 5.56 & 5.56 & 38.89 & 50.00 & 66.67 & 83.33 & 8.87 & 20.16 & 32.66 & 37.90 & 49.60 & 58. \\
\hline XICE 46 & 6.77 & 21.69 & 45.17 & 58.88 & 73.68 & 81.80 & 11.11 & 33.33 & 44.44 & 55.56 & 72.22 & 83.33 & 3.14 & 13.03 & 1.65 & 4.36 & 99.70 & 59.9 \\
\hline AA 22] & 43 & 2.15 & 9.01 & 17.17 & 28.76 & 39.06 & 0 & 5.56 & 33.33 & 38.89 & 66.67 & 2.22 & 10.48 & 16.53 & 27.02 & 6.69 & 53.63 & 60.48 \\
\hline $\mathrm{A}^{4}-2$ & 72 & 16 & & 35 & & & 0 & 5.56 & & 44.44 & & & & & & 61.69 & 71.77 & 83.4 \\
\hline phop+CRAFT & 15 & 9.44 & 27.04 & 38.63 & 48.07 & 55.36 & 11.11 & 22.22 & 38.89 & 55.56 & 77.78 & 83.33 & 23.79 & 33.87 & 42.74 & 47.58 & 55.65 & 59.68 \\
\hline JSTL- & .92 & 4 & 8 & 46 & 1 & 9 & 33 & 33.33 & 33 & 55.56 & .56 & .67 & 10 & 59.52 & 71.43 & 6.19 & 88. & 92.8 \\
\hline Res & & 00 & & 96.92 & 46 & 23 & 44 & 56 & & & 78 & 8 & 90 & 81 & .48 & .24 & & \\
\hline Sko 43] & 15 & 78.46 & 94.62 & 96.15 & 99.23 & 99.23 & 44 & 55.56 & 55. & 55.56 & 55.56 & 77.78 & 1.29 & 71.43 & 88.10 & 0.48 & 92.8 & 95. \\
\hline DeepFo & 34.62 & 78.46 & 94.63 & 96.92 & 96.92 & 99.23 & 14 & 55.56 & 55. & 55.56 & 66.67 & 77.78 & 1.29 & 76.19 & 90. & 5.2 & 5. & 95.2 \\
\hline & & 02.01 & 0.92 & 98.46 & 3 & 100 & D.jo & 00.5 & & 00.0 & 77.78 & $7 . .7$ & 00.0 & & $92 .{ }_{4}$ & 95.2 & 0.2 & 95.24 \\
\hline E & \multicolumn{18}{|c|}{$\overline{\overline{\text { Verification }}}$} \\
\hline t-LRI & 54 & & .44 & 49 & 10 & 87.63 & & .15 & 51.82 & 67.56 & 73.54 & 9.1 & & 37.40 & 18.98 & 8.83 & 70.76 & 90.1 \\
\hline 6] & .64 & 61.58 & 84.87 & 90.68 & 96.86 & 97.21 & 33.33 & 36.11 & 55.56 & 55.56 & 72.22 & 88.89 & 8.63 & 48.94 & 71.89 & 81.64 & 89.71 & 97.43 \\
\hline 2 & & & & 77 & 9 & & & & & & 97.22 & 100 & & 45.73 & & 85 & 87.40 & 91.99 \\
\hline $\mathrm{QDA} 22$ & 25.32 & 59.10 & 81.98 & .96 & 92.96 & 94.84 & 56 & 11 & 80.56 & 88.89 & 88.89 & 88.89 & .72 & 79 & 77.78 & 6.90 & 94.44 & .03 \\
\hline $\left.\mathrm{FT}^{6} 6\right]$ & 31.75 & & 84.09 & 42 & 93.55 & 96.30 & 50.00 & 72.22 & 100 & & 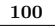 & 100 & 39 & 27 & 38 & 9.58 & 68 & 99.18 \\
\hline 38 & 47.23 & & 92 & 73 & .73 & & & & 59.26 & & 84 & 2.72 & & 18 & & 2.1 & 5 & 948 \\
\hline ResNet-50 $\overline{\Gamma 2}$ & 82.26 & 95.86 & 54 & 38 & 99.58 & 99 & 44.44 & 50.00 & 72.22 & 77.78 & 83 & 33 & 76.19 & 91.67 & & 24 & .24 & 5.24 \\
\hline SRO 43] & & & & 96 & 99.1 & & 4 & 6.1 & 72.22 & 77 & & 3 & 73.81 & & & 2 & 95.24 & 95.24 \\
\hline DeepFool 28] & 82.26 & 95.86 & 95.86 & 98.96 & 99.17 & 99.58 & 44.44 & 61.11 & 72.22 & 77.78 & 83.33 & 83.33 & 75.61 & 91.67 & 95.2 & 95.2 & 95.24 & 95.24 \\
\hline & 84.00 & & & & & 80.00 & 44.44 & 61.11 & 77.78 & 77.78 & 83.33 & & & & & & 7.15 & 97.15 \\
\hline
\end{tabular}

In pre-training, we used stochastic gradient descent with momentum 0.9. The learning rate was 0.1 at the beginning and multiplied by 0.1 every 10 epochs. Then, the adversarial part of APN was trained using ADAM optimizer [16] with parameters $\beta_{1}=0.5$ and $\beta_{2}=0.99$. Using the target dataset for evaluation, the person discriminator $D_{p}$ and generator $G$ were pre-trained for 20 epochs. Then, the target discriminator $D_{t}$ together with the person discriminator $D_{p}$, and the generator $G$ were trained jointly for $k_{1}=15$ epochs, where $G$ is optimized twice in each iteration to prevent losses of discriminators from going to zero. Finally, the feature extractor was trained again for $k_{2}=20$ epochs with a lower learning rate starting from 0.001 and multiplied by 0.1 every 10 epochs. The above procedure was executed repeatedly as an adversarial process.

\subsection{Comparison with Open-world Re-id Methods}

Open-world re-id is still under studied, and t-LRDC 42 and XICE 46 are two represented existing methods designed for the open-world setting in person re-id. The results are reported in Table 1 1 Our APN outperformed t-LRDC and XICE in almost all cases, and the margin is especially large on CUHK03. Compared to t-LRDC and XICE, our APN is an end-to-end learning framework and takes adversarial learning into account for feature learning, so that APN is more tolerant to the attack of samples of non-target people.

\subsection{Comparison with Closed-world Re-id Methods}

We compared our method with related popular re-id methods developed for closed-world person re-identification. We mainly evaluated ResNet-50 [12], XQDA 22], CRAFT 6], DCGAN+LSRO [43, and JSTL-DGD [38] for comparison. These methods were all evaluated by following the same setting as our APN. As 
Table 2: Different generated imposter sources

\begin{tabular}{|c|c|c|c|c|c|c|c|c|c|c|c|c|c|c|c|c|c|c|}
\hline Dataset & \multicolumn{6}{|c|}{ Market-1501 } & \multicolumn{6}{|c|}{ CUHK01 } & \multicolumn{6}{|c|}{ CUHK03 } \\
\hline FTR & $0.1 \%$ & $1 \%$ & $5 \%$ & $10 \%$ & $20 \%$ & $30 \%$ & $0.1 \%$ & $1 \%$ & $5 \%$ & $10 \%$ & $20 \%$ & $30 \%$ & $0.1 \%$ & $1 \%$ & $5 \%$ & $10 \%$ & $20 \%$ & $30 \%$ \\
\hline Evaluation & \multicolumn{18}{|c|}{ Set Verification } \\
\hline APN & 43.8 & 82.31 & 96 & - & 99.23 & 00 & 55.5 & & 55.56 & 66.67 & 77.7 & 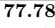 & 66.6 & 78.57 & 2.8 & . & 95.24 & 5.24 \\
\hline PN w/o $D_{t}$ & .92 & 79.23 & 93.85 & 97.69 & 99.23 & 99.23 & 4.44 & 55.56 & 55.56 & 55.56 & 55.56 & 66.67 & 61.90 & 73.81 & 88.10 & 90.48 & 92.86 & 95.2 \\
\hline APN w/o $D_{p}$ & .38 & 78.46 & 93.85 & 95.38 & 99.23 & 99.23 & 44.44 & 55.56 & 55.56 & 55.56 & 55.56 & 66.67 & 61.90 & 71.43 & 88.10 & 92.86 & 95.24 & 95.24 \\
\hline PN w/o $W S$ & .46 & 65.38 & 1.62 & 89.23 & 96.15 & 96.15 & 44.44 & 55.56 & 55.56 & 55.56 & 55.56 & 66.67 & 57.14 & 73.81 & 83.33 & 90.48 & 92.86 & 92.86 \\
\hline No Imposter & 34.62 & 80.00 & 93.85 & 96.92 & 98.46 & 99.23 & 44.44 & 55.56 & 55.56 & 55.56 & 77.78 & $\mathbf{7 7 . 7 8}$ & 61.90 & 73.81 & 90.48 & 95.24 & 9.2 & 95.24 \\
\hline Evalu & \multicolumn{18}{|c|}{ Verific } \\
\hline $\mathrm{AI}$ & 84.00 & 6.7 & 8.6 & 9.5 & 99.58 & & & 1 & 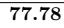 & 77.7 & 83.3 & & 9.5 & & 5.2 & & & 97.1 \\
\hline APN w/o $D_{t}$ & 2.92 & 95.40 & 13 & 98.75 & 99.58 & 99. & 44.44 & 55.56 & 77.78 & 77.78 & 83.33 & 83.33 & 73.81 & 0.48 & 95.2 & 95. & 195.24 & 95.24 \\
\hline$D_{p}$ & 2.26 & 95.06 & 13 & 98 & 99 & 95 & 44.44 & 50.00 & 77.78 & 77 & 83 & 8 & 72.62 & 48 & 91.67 & 9 & 24 & 95.24 \\
\hline & & & & & & 97. & & & 77.78 & 77 & & & 69.05 & 84.52 & 94 & 95 & 19 & 95.24 \\
\hline mposter & 2.26 & .86 & .54 & & 9.5 & 0 & .4 & .00 & 72.22 & $\mathbf{7 7 . 7 8}$ & 83.3 & 3.33 & 76.19 & 91.67 & 95.2 & 95.24 & 5.24 & 5.24 \\
\hline
\end{tabular}

shown in Table 1 these approaches optimal for closed-world scenario cannot well adapt to the open-world setting. In the cases of Market-1501 and CUHK03, the proposed APN achieved overall better performance, especially when tested on Set Verification and when FTR is $0.1 \%$ as compared to the others. On Market-1501, APN obtained 7.7 more matching rate than the second place DCGAN+LSRO, which is also an application of GAN in re-id problem, when FTR is $0.1 \%$ on Set Verification, and as well outperformed DCGAN+LSRO on all conditions on Individual Verification. Although deep learning methods may cause overfitting problem on CUHK01 dataset, which is shown in Individual Verification of CUHK01 where hiphop+CRAFT had the best performance, APN outperformed all other deep learning methods on all conditions of CUHK01. When FTR is $0.1 \%$ on CUHK01 Set Verification, APN gained 11.12 more matching rate as compared to DCGAN+LSRO and 5.56 matching rate more when FTR is $5 \%$ on Individual Verification. The compared closed-world models were designed with the assumption that the same identities hold between gallery and probe sets, while the relation between target and non-target people is not modelled. Meanwhile our APN is designed for the open-world group-based verification for discriminating target from non-target people.

\subsection{Comparison with Related Adversarial Generation}

We compared our model with fine-tuned ResNet-50 with adversarial samples generated by DeepFool [28], which is also a method using extra generated samples. DeepFool produced adversarial samples to fool the network by adding noises computed by gradients. As shown in Table 1, our APN performed much better than DeepFool on all conditions. DeepFool cannot adapt to open-world re-id well because the adversarial samples generated are produced with a separate learning from the classifier learning and thus the relation between the generated samples and target set is not modelled for group-based verification, while in our APN we aim to generate target-like samples so as to make adversarial learning facilitate learning better features. 


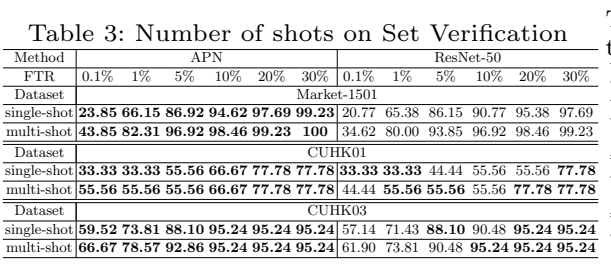

Table 4: Number of shots on Individual Verification
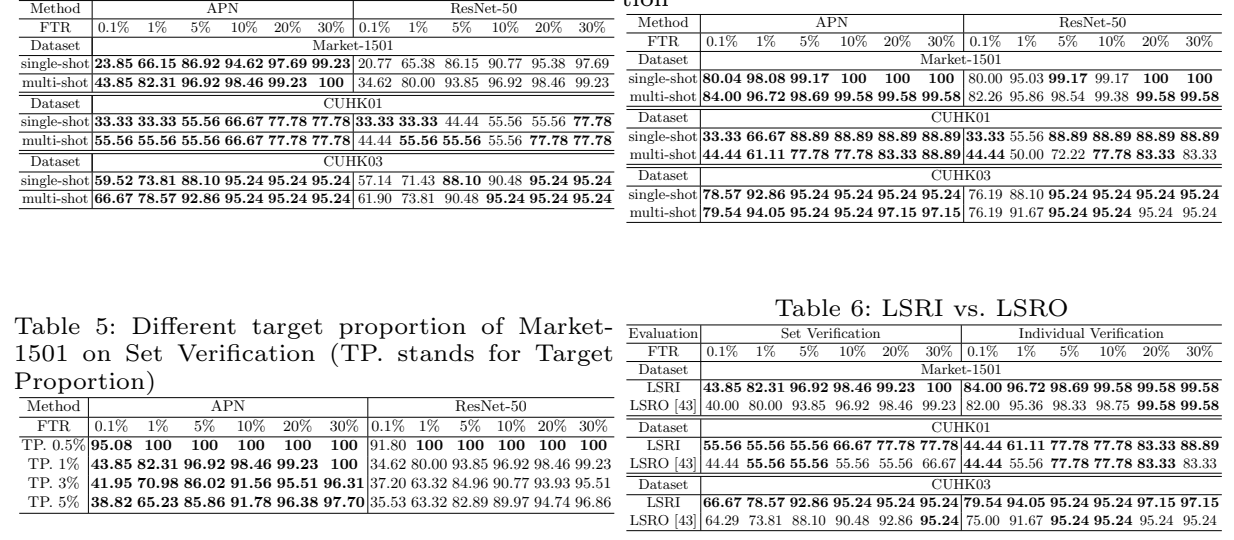

\subsection{Further Evaluation of Our Method}

Effect of Generated Imposters. It can be observed that, training with the imposters generated by APN can achieve large improvement as compared to the case without it, because these imposters are target-like and can improve the discriminating ability of the features. The results of baseline without any generated imposters are shown in the rows indicated by No Imposters in Table 2 . In details, on Set Verification, APN outperformed 9.23 matching rate on Market1501, 11.12 more matching rate on CUHK01 and 4.77 on CUHK03 when FTR is $0.1 \%$. On Individual Verification, APN also has better performance on all cases. Effect of Weight Sharing. The weight sharing between the target discriminator and the feature extractor aims to ensure that the generator can learn from the feature extractor and generate more target-like attack samples. Without the sharing, there is no connection between generation and feature extraction. Taking Set Verification on Market for instance, ours degrades from $82.31 \%$ to $65.38 \%$ (no sharingindicated by "APN w/o WS") when FTR=1\% in Table 2

Effect of Person Discriminator and Target Discriminator. Our APN is based on GAN consisting of generator, person discriminator $D_{p}$ and target discriminator $D_{t}$. To further evaluate them, we compared with APN without person discriminator (APN w/o $D_{p}$ ) and APN without target discriminator (APN w/o $\left.D_{t}\right)$. APN without target discriminator can be regarded as two independent components DCGAN and feature extraction network. To fairly compare these cases, LSRI was also applied as in APN for the generated samples. The results are reported in Table 2. It is obvious that our full APN is the most effective one among the compared cases. Sometimes generating imposters by APN without person discriminator $D_{p}$ or target discriminator $D_{t}$ even degrade the performance as compared to the case of no imposter. When target discriminator is discarded, although person-like images can be generated, they are not similar to target people and thus are not serious attacks to the features for group-based verification. In the case without person discriminator, the generator even fails to 
generate person-like images (see Fig. 3) so that the performance is also degraded in most cases. This indicates that the person discriminator plays an important role in generating person-like images, and the target discriminator is significant for helping the generator to generate better target-like imposters, so that the feature extractor can benefit more from distinguishing these imposters.

LSRI vs LSRO. We verified that the modification of LSRO, namely LSRI in Eq. (6) is more suitable for optimizing the open-world re-id. The performance of comparing our LSRI with the original LSRO is reported in Table6. It shows that the feature extractor is more likely to correctly discriminate target people under the same FTR using LSRI on. It is proved that our modification LSRI is more appropriate for open-world re-id scenario, since the imposters are allocated equal probabilities of being non-target for group-based towards modelling, so they are more likely to be far away from target person samples, leading to more discriminative feature representation for target people, while in LSRO, the imposters are allocated equal probabilities of being non-target as well as target. Effect of target proportion. The evaluation results on different target proportion are reported in Table 5 . We used different percentages of people marked as target. This verification was conducted on Market-1501, and we used original ResNet-50 for comparison. While TTR declines with the growth of target proportion due to more target people to verify, our APN can still outperformed the original ResNet-50 in all cases.

Effect of the Number of Shots. The performance under multi-shot and single-shot settings were also compared in our experiments. For multi-shot setting, we randomly selected two images of each target person as gallery set, while for single-shot setting, we only selected one. As shown in Table 3 and Table 4 , on both single-shot and multi-shot settings, our APN outperformed ResNet-50 on all conditions of Market-1501, CUHK01, and CUHK03. Especially on Set Verification, for CUHK01, when FTR is 10\%, APN outperformed ResNet-50 11.11\% under both single-shot and multi-shot settings.

\section{Conclusion}

For the first time, we demonstrate how adversarial learning can be used to solve the open-world group-based person re-id problem. The introduced adversarial person re-id enables a mutually related and cooperative progress among learning to represent, learning to generate, learning to attack, and learning to defend. In addition, this adversarial modelling is also further improved by a label smoothing regularization for imposters under semi-supervised learning.

\section{Acknowledgment}

This work was supported partially by the National Key Research and Development Program of China (2016YFB1001002) and the NSFC(61522115, 61472456), Guangdong Programme (2016TX03X157), and the Royal Society Newton Advanced Fellowship (NA150459). The corresponding author for this paper is WeiShi Zheng. 


\section{References}

1. Ahmed, E., Jones, M.J., Marks, T.K.: An improved deep learning architecture for person re-identification. In: CVPR. IEEE Computer Society (2015)

2. Baltieri, D., Vezzani, R., Cucchiara, R.: 3dpes: 3d people dataset for surveillance and forensics. In: Proceedings of the 1st International ACM Workshop on Multimedia access to 3D Human Objects. pp. 59-64. Scottsdale, Arizona, USA (Nov 2011)

3. Bedagkar-Gala, A., Shah, S.K.: A survey of approaches and trends in person reidentification. Image Vision Comput. 32(4), 270-286 (2014)

4. Cancela, B., Hospedales, T.M., Gong, S.: Open-world person re-identification by multi-label assignment inference. In: Valstar, M.F., French, A.P., Pridmore, T.P. (eds.) BMVC. BMVA Press (2014)

5. Chen, Y.C., Zheng, W.S., Lai, J.H., Yuen, P.C.: An asymmetric distance model for cross-view feature mapping in person reidentification. IEEE Trans. Circuits Syst. Video Techn. 27(8), 1661-1675 (2017)

6. Chen, Y.C., Zhu, X., Zheng, W.S., Lai, J.H.: Person re-identification by camera correlation aware feature augmentation. CoRR abs/1703.08837 (2017)

7. Cheng, D., Gong, Y., Zhou, S., Wang, J., Zheng, N.: Person re-identification by multi-channel parts-based cnn with improved triplet loss function. In: CVPR. IEEE Computer Society (2016)

8. Deng, W., Zheng, L., Ye, Q., Kang, G., Yang, Y., Jiao, J.: Image-image domain adaptation with preserved self-similarity and domain-dissimilarity for person reidentification. In: CVPR. p. 6. IEEE Computer Society (2018)

9. Ding, S., Lin, L., Wang, G., Chao, H.: Deep feature learning with relative distance comparison for person re-identification. CoRR abs/1512.03622 (2015)

10. Farenzena, M., Bazzani, L., Perina, A., Murino, V., Cristani, M.: Person reidentification by symmetry-driven accumulation of local features. In: CVPR. IEEE Computer Society (2010)

11. Gong, S., Cristani, M., Yan, S., Loy, C.C. (eds.): Person Re-Identification. Advances in Computer Vision and Pattern Recognition, Springer (2014)

12. He, K., Zhang, X., Ren, S., Sun, J.: Deep residual learning for image recognition. In: CVPR. IEEE Computer Society (2016)

13. Hirzer, M., Beleznai, C., Roth, P.M., Bischof, H.: Person re-identification by descriptive and discriminative classification. In: SCIA. Springer (2011)

14. Hirzer, M., Roth, P.M., Kstinger, M., Bischof, H.: Relaxed pairwise learned metric for person re-identification. In: ECCV. Lecture Notes in Computer Science (2012)

15. Kawanishi, Y., Wu, Y., Mukunoki, M., Minoh, M.: Shinpuhkan2014: A multicamera pedestrian dataset for tracking people across multiple cameras

16. Kingma, D.P., Ba, J.: Adam: A method for stochastic optimization. CoRR abs/1412.6980 (2014)

17. Kviatkovsky, I., Adam, A., Rivlin, E.: Color invariants for person reidentification. IEEE Trans. Pattern Anal. Mach. Intell. 35(7), 1622-1634 (2013)

18. Kstinger, M., Hirzer, M., Wohlhart, P., Roth, P.M., Bischof, H.: Large scale metric learning from equivalence constraints. In: CVPR. IEEE Computer Society (2012)

19. Li, W., Zhao, R., Wang, X.: Human reidentification with transferred metric learning. In: ACCV (2012)

20. Li, W., Zhao, R., Xiao, T., Wang, X.: Deepreid: Deep filter pairing neural network for person re-identification. In: CVPR. IEEE Computer Society (2014) 
21. Li, W., Zhao, R., Xiao, T., Wang, X.: Deepreid: Deep filter pairing neural network for person re-identification. In: CVPR (2014)

22. Liao, S., Hu, Y., Zhu, X., Li, S.Z.: Person re-identification by local maximal occurrence representation and metric learning. In: CVPR. pp. 2197-2206. IEEE Computer Society (2015)

23. Liu, C., Gong, S., Loy, C.C.: On-the-fly feature importance mining for person reidentification. Pattern Recognition 47(4), 1602-1615 (2014)

24. Lu, J., Issaranon, T., Forsyth, D.A.: Safetynet: Detecting and rejecting adversarial examples robustly. CoRR abs/1704.00103 (2017)

25. Ma, B., Su, Y., Jurie, F.: Bicov: a novel image representation for person reidentification and face verification. In: BMVC (2012)

26. Ma, B., Su, Y., Jurie, F.: Covariance descriptor based on bio-inspired features for person re-identification and face verification. Image Vision Comput. 32(6-7), 379-390 (2014). https://doi.org/10.1016/j.imavis.2014.04.002

27. Mignon, A., Jurie, F.: Pcca: A new approach for distance learning from sparse pairwise constraints. In: CVPR. IEEE Computer Society (2012)

28. Moosavi-Dezfooli, S.M., Fawzi, A., Frossard, P.: Deepfool: A simple and accurate method to fool deep neural networks. In: CVPR. IEEE Computer Society (2016)

29. Papernot, N., McDaniel, P.D., Jha, S., Fredrikson, M., Celik, Z.B., Swami, A.: The limitations of deep learning in adversarial settings. CoRR abs/1511.07528 (2015)

30. Radford, A., Metz, L., Chintala, S.: Unsupervised representation learning with deep convolutional generative adversarial networks. CoRR abs/1511.06434 (2015)

31. Subramaniam, A., Chatterjee, M., Mittal, A.: Deep neural networks with inexact matching for person re-identification. In: Lee, D.D., Sugiyama, M., von Luxburg, U., Guyon, I., Garnett, R. (eds.) NIPS (2016)

32. Szegedy, C., Zaremba, W., Sutskever, I., Bruna, J., Erhan, D., Goodfellow, I.J., Fergus, R.: Intriguing properties of neural networks. CoRR abs/1312.6199 (2013)

33. Tao, D., Jin, L., Wang, Y., Yuan, Y., Li, X.: Person re-identification by regularized smoothing kiss metric learning. IEEE Trans. Circuits Syst. Video Techn. 23(10), 1675-1685 (2013)

34. Wang, H., Zhu, X., Xiang, T., Gong, S.: Towards unsupervised open-set person re-identification. In: ICIP. IEEE (2016)

35. Wang, T., Gong, S., Zhu, X., Wang, S.: Person re-identification by discriminative selection in video ranking. IEEE transactions on pattern analysis and machine intelligence 38(12), 2501-2514 (2016)

36. Wu, L., Shen, C., van den Hengel, A.: Personnet: Person re-identification with deep convolutional neural networks. CoRR abs/1601.07255 (2016)

37. Wu, S., Chen, Y.C., Li, X., Wu, A., You, J., Zheng, W.S.: An enhanced deep feature representation for person re-identification. CoRR abs/1604.07807 (2016)

38. Xiao, T., Li, H., Ouyang, W., Wang, X.: Learning deep feature representations with domain guided dropout for person re-identification. In: CVPR. IEEE Computer Society (2016)

39. Zheng, L., Shen, L., Tian, L., Wang, S., Wang, J., Tian, Q.: Scalable person reidentification: A benchmark. In: ICCV (2015)

40. Zheng, W.S., Gong, S., Xiang, T.: Person re-identification by probabilistic relative distance comparison. In: CVPR. IEEE Computer Society (2011)

41. Zheng, W.S., Gong, S., Xiang, T.: Transfer re-identification: From person to setbased verification. In: CVPR. IEEE Computer Society (2012)

42. Zheng, W.S., Gong, S., Xiang, T.: Towards open-world person re-identification by one-shot group-based verification. IEEE Trans. Pattern Anal. Mach. Intell. 38(3), 591-606 (2016) 
43. Zheng, Z., Zheng, L., Yang, Y.: Unlabeled samples generated by gan improve the person re-identification baseline in vitro. CoRR abs/1701.07717 (2017)

44. Zhong, Z., Zheng, L., Zheng, Z., Li, S., Yang, Y.: Camera style adaptation for person re-identification. In: CVPR. pp. 5157-5166. IEEE Computer Society (2018)

45. Zhu, J., Zeng, H., Liao, S., Lei, Z., Cai, C., Zheng, L.: Deep hybrid similarity learning for person re-identification. CoRR abs/1702.04858 (2017)

46. Zhu, X., Wu, B., Huang, D., Zheng, W.S.: Fast open-world person reidentification. IEEE Transactions on Image Processing PP(99), 1-1 (2017). https://doi.org/10.1109/TIP.2017.2740564 